\title{
Ahora que Estas Pelona. Frida Kahlo : l'Ambigüité du genre
}

Ahora que Esta Pelona, Frida Kahlo: gender ambiguity

Julie Crenn

\section{OpenEdition}

Journals

Édition électronique

URL : http://journals.openedition.org/clio/10836

DOI : $10.4000 /$ clio. 10836

ISSN : 1777-5299

Éditeur

Belin

Édition imprimée

Date de publication : 31 décembre 2012

Pagination : 191-201

ISSN : 1252-7017

Référence électronique

Julie Crenn, «Ahora que Estas Pelona. Frida Kahlo : l'Ambigüité du genre », Clio. Femmes, Genre,

Histoire [En ligne], 36 | 2012, mis en ligne le 31 décembre 2014, consulté le 21 décembre 2020. URL:

http://journals.openedition.org/clio/10836 ; DOI : https://doi.org/10.4000/clio.10836 


\section{Ahora que Estas Pelona. Frida Kahlo : l'Ambigüité du genre}

Julie CRENN

L'œuvre de Frida Kahlo (1907-1954) est aujourd'hui reconnue internationalement par les institutions et le grand public; un succès unanime qui s'explique par la singularité de son style, les choix thématiques et une personnalité largement relayée par le biais de biographies diverses et d'expositions à travers le monde. André Breton a écrit en 1938: «L'art de Frida Kahlo est un ruban autour d'une bombe $»^{1}$. Un art coloré et souvent maladroitement jugé naif, qui porte en lui un engagement qu'il est nécessaire d'analyser. En explorant une autoreprésentation compulsive, Frida Kahlo a testé et transgressé les limites imposées par la société patriarcale du Mexique des années 1930-1950, ainsi que celles imposées par l'école des Beaux-Arts de Mexico, l'histoire de l'art et la religion. Nous avons choisi d'axer notre réflexion sur une œuvre de 1940. Un autoportrait que nous souhaitons mettre en lumière car il révèle une conscience protoféministe, un attrait pour l'ambiguité sexuelle et une réflexion précoce sur la dichotomie des genres.

La question du genre s'est imposée à Frida Kahlo dans sa vie privée comme dans son œuvre. Elle arborait à la fois une allure et un comportement très féminins, et parallèlement affichait une attitude plus masculine et virile. Du moins une attitude qui n'était pas associée à l'image que la société traditionnelle mexicaine des années 1930-1940 se faisait des femmes. Après son mariage avec Diego Rivera, elle adopte la robe Tehuana, un symbole important puisqu'elle incarne la femme forte au sein d'une microsociété matriarcale indienne. Frida

1 Breton $1965: 144$. 
Kahlo apparaissait en public en robes Tehuana, qu'elle soit au Mexique, aux États-Unis ou à Paris. C'était une manière pour elle d'affirmer son identité mexicaine dont elle était extrêmement fière. Les robes correspondaient aussi aux costumes de son personnage toujours flamboyant et extravagant. À l'heure de la révolution au Mexique, les Tebuanas, robes issues de la société matriarcale de Tehuantepec, étaient devenues des icônes de la mexicanidad (mouvement populaire porté par les idées révolutionnaires, incitant à un retour aux racines culturelles mexicaines et indiennes). La robe symbolise donc la force et l'indépendance, car la région de Tehuantepec a su rester libre face aux lois machistes et coloniales. Lorsqu'elle était portée par Frida Kahlo, la robe Tehuana traduisait non seulement son identité culturelle mais aussi son indépendance en tant que femme et en tant qu'artiste. Elle instaurait ainsi une dissonance entre son apparence féminine et une façon de se comporter qui était associée à la sphère masculine. Elle parlait et écrivait de manière extrêmement vulgaire, elle buvait et fumait à outrance, elle employait et inventait des jurons, qui, à cette époque, étaient uniquement utilisés par les hommes.

En 1940, elle réalise Autorretrato con Pelo Cortado ${ }^{2}$, où elle se représente assise sur une chaise de paille jaune, au centre d'une pièce aux dimensions apparemment infinies. La partie haute de son corps est placée sur un fond gris qui rappelle le ciel nuageux présent dans plusieurs de ses œuvres. La partie basse de son corps se détache sur un fond brun, le sol de la pièce, comme recouvert de terre. Frida Kahlo apparaît dans un espace intemporel. Elle se présente assise de trois quarts, face au spectateur qu'elle regarde fixement en détournant légèrement la tête vers la gauche. Les traits de son visage au teint pâle paraissent tirés et fatigués. Elle est vêtue d'un costume gris sombre, d'une chemise pourpre et de chaussures noires à talons. À son oreille, une boucle est suspendue. Le sol et la chaise sont jonchés des mèches noires de sa chevelure. Entre ses jambes, elle tient dans sa main gauche ses cheveux coupés et dans sa main droite une paire de ciseaux. Au sommet de la toile sont inscrites les paroles d'une chanson populaire mexicaine : "Mira que si te quise, fue por el pelo, Ahora

2 http://www.moma.org 
que estas pelona, ya no te quieros » (Tu vois, si je t'aimais, c'était pour tes cheveux, maintenant que tu es chauve, je ne t'aime plus). Des paroles qui ont immédiatement conduit certains auteurs vers une interprétation strictement biographique, en la reliant à la rupture entre Frida Kahlo et Diego Rivera. La biographe officielle de l'artiste, Hayden Herrera, estime que l'œuvre baigne dans une «atmosphère vengeresse ». Elle précise que Kahlo aurait menacé son époux de couper ses cheveux s'il ne mettait pas fin à sa liaison avec une autre femme. Une menace mise à exécution puisqu'il a refusé de céder au chantage. Herrera pense également que le costume gris que porte l'artiste pourrait être celui de Rivera, étant donné son ampleur. Elle ajoute enfin que «Frida se montre comme l'inoubliable incarnation de la colère et de la féminité blessée $»^{3}$. Le fait d'inscrire l'œuvre dans une optique de vengeance futile et illusoire « renforce des codes de genre sans évaluer à quel point la peinture peut les employer comme un moyen de critiquer ou subvertir la prescription sociale moralisante $»^{4}$. Le fait même de penser qu'en se présentant ainsi elle s'adressait uniquement à son compagnon est dévaluant et réducteur par rapport à son statut d'artiste, cela impliquerait qu'elle ne prenait pas en considération le regard du spectateur.

\section{Masquer la réalité}

Les autoportraits de Frida Kahlo peuvent être envisagés comme des masques. Les masques d'un personnage qu'elle a façonné toute sa vie : Frida. En public l'artiste était rayonnante, portant des robes spectaculaires ; en privé, elle écrivait ceci : «Maintenant je me sens si pourrie et si seule qu'il me semble que personne au monde a souffert comme moi, mais bien sûr ce sera différent j'espère dans quelques mois $»^{5}$. Le masque social occultait un désarroi psychologique et physique. La lecture de son dossier médical nous apprend qu'à partir de 1939, elle buvait « pratiquement une bouteille de Cognac par jour » pour atténuer ses douleurs. Une situation qui l'a menée à un geste

\footnotetext{
Herrera 1996 : 370-372.

Lindauer 1999: 44.

Herrera 1996 : 358. [Lettre à Nikolas Muray, datée du 13 octobre 1939].
} 
crucial dans notre analyse, celui de se couper les cheveux ${ }^{6}$. Une récidive, car en 1934, après avoir appris que Rivera lui était infidèle avec sa propre sœur, Kahlo avait coupé sa longue chevelure. Une action symbolique forte traduisant la volonté de supprimer ce qui caractérise une partie de sa féminité.

L'œuvre procède alors à une déconstruction visuelle du concept de féminité. Sarah M. Lowe explique que la peinture «explore la construction sociale de la femme [...] elle ne met pas en avant une analyse de sa propre identité, mais une exégèse de la définition culturelle de la féminité $»^{7}$. Contrairement à ce qu'avance Herrera, qui y voit un violent rejet de sa féminité puisque Kahlo se présente au spectateur habillée comme un homme (Herrera fait abstraction de la boucle d'oreille et des talons hauts), Margaret Lindauer estime qu'elle «a plutôt combiné les attributs féminins et masculins, se situant dans une catégorie énigmatique entre les stéréotypes féminins et masculins ${ }^{8}$. Ainsi, Frida Kahlo s'est débarrassée des "pièges sociaux de la féminité » qui sont les cheveux longs, la robe et la posture. Elle a choisi d'accentuer sa part masculine. Elle est assise les jambes écartées, défiant le regard du spectateur, une attitude rigide et stoïque inhabituelle pour une femme. Deux vestiges féminins subsistent, la boucle d'oreille et les chaussures noires à talons. Deux symboles confirmant une identité sexuelle trouble. De plus, il n'est pas évident que le costume soit celui de Rivera, car grâce aux divers portraits photographiques, nous savons que depuis son adolescence Kahlo arborait des vêtements masculins. Une photographie datée de 1947, montre l'artiste vêtue d'un costume sombre et large, proche de celui d'Autorretrato con Pelo Cortado ${ }^{9}$. Elle jette volontairement un trouble, l'œuvre présentnt une possibilité de libération des femmes de la tyrannie du genre et du joug du phallocentrisme. Une libération qui permettrait aux femmes d'accéder aux privilèges sociaux qui leur sont « naturellement » interdits.

Tibol $1993: 14$

Lowe 1991 : 59-61.

Lindauer $1999: 15$.

Poniatowska 1992 : 73. 
À la suite des deux grandes ruptures du couple (en 1934 et 1939), Kahlo avait coupé ses cheveux et portait le pantalon en signe de protestation: une forme de rituel impliquant une abolition de son personnage et une renaissance personnelle. L'artiste véhicule ainsi un message, celui d'une révolte individuelle. Il faut également signaler que dans les années trente et quarante, il n'était pas aisé pour une artiste d'intégrer les cercles artistiques, du fait de son sexe encore considéré comme inférieur. De nombreuses artistes ont adopté une apparence ou un comportement volontairement masculin afin de se « fondre » dans le système dominant ${ }^{10}$. Dans ce sens, il est intéressant d'observer que le fait de laisser pousser ses cheveux pour une femme est un signe de passivité, tandis que si elle décide de les couper, elle prend une part active dans le processus naturel tout en transgressant les conventions culturelles de l'époque. Effectivement, Frida Kahlo s'est insurgée contre les prescriptions sociales. Ainsi la rupture est symbolisée par la paire de ciseaux et la tresse coupée que l'artiste tient dans sa main droite entre ses jambes.

Le spectateur associe naturellement cet outil aux ciseaux chirurgicaux qui servent, entre autres, à couper le cordon ombilical ou à la castration. Si la représentation de Kahlo est envisagée du côté masculin, les ciseaux apparaissent comme un symbole phallique placé entre les jambes de l'artiste. Si elle est considérée du côté féminin, ils font directement référence à la notion freudienne de la femme naturellement castrée. La paire de ciseaux fait aussi allusion à la peur qu'ont les hommes des castratrices, c'est-à-dire les femmes de pouvoir ${ }^{11}$. Le concept freudien de l'angoisse de la castration est étroitement associé au complexe d'Edipe, la reconnaissance du père,

10 Nous pouvons citer les exemples de Rosa Bonheur, Hannah Höch ou encore Claude Cahun. De plus les femmes artistes et écrivains ont très souvent adopté des pseudonymes masculins afin de ne subir aucune discrimination sexuelle, comme George Sand ou Charlotte Brontë.

11 Lindauer 1999 : 45. Margaret Lindauer cite Jane Flax qui décrit la castration : «ce qui est craint avec la "castration" c'est de manquer ou de perdre le pénis - c'està-dire, être ou devenir une femme... Une telle absence entraîne nécessairement l'exclusion du monde privilégié masculin, de "l'association constante avec les hommes", sur lesquels chacun est "dépendant" pour accomplir tout objectif culturel ». 
par conséquent du phallus. Il s'agit alors d'une étape fondamentale de la psychanalyse dans le développement sexuel du jeune enfant. Chez la jeune fille, la reconnaissance est différente puisqu'elle prend conscience que la castration a eu lieu avant sa naissance ; surviennent ensuite un état de manque et une recherche de «l'objet imaginaire $»^{12}$. Frida Kahlo critique le poids social démesuré que détiennent les hommes en faisant ironiquement allusion au phallus, ou à l'abandon de celui-ci par les femmes, le phallus étant l'élément biologique et symbolique incarnant le pouvoir masculin.

\section{Modèles littéraires}

À ce moment précis de sa vie, Frida Kahlo refuse l'association réductrice à un corps souffrant ou à une simple victime du pouvoir patriarcal. Elle s'est probablement inspirée de George Sand, dont elle admirait la vie et l'œuvre, puisque Sand avait également coupé ses cheveux après le départ d'Alfred de Musset. En témoigne un portrait réalisé par Eugène Delacroix, où l'auteure apparait les cheveux courts et vêtue d'un costume d'homme. Ce portrait, ainsi que le geste à la fois désespéré et défiant de George Sand l'ont probablement inspiré ${ }^{13}$. Un décryptage attentif de la bibliothèque de l'artiste nous amène vers un exemplaire de l'autobiographie de Sor Juana Inès de la Cruz, Repuesta a Sor Philothea (Réponse à Scur Philothea) $1691^{14}$. Un texte dans lequel, celle-ci raconte que pour se punir de sa lenteur dans son apprentissage du latin, elle se coupait tous les jours plusieurs mèches de cheveux. Un acte de punition et de remotivation. Comme la religieuse, Frida Kahlo semblait gagner de la puissance et du courage en coupant ses cheveux. Elle se punissait pour ses faiblesses par rapport au discours dominant. Gannit Ankori nous apprend en outre qu'à la même période l'artiste lisait probablement l'Autobiographie de

12 Freud 1926.

13 On sait aujourd'hui que l'artiste se passionnait pour les biographies de femmes, ce qui selon Ankori montrait la "construction d'une conscience féministe". En ce qui concerne George Sand, Frida Kahlo possédait dans sa bibliothèque Winwar, Frances (1945). The Life of the Heart: George Sand and Her Times, ainsi que les trois volumes de Raymond Escholier (1926), Delacroix : Peintre, Graveur, Écrivain.

14 De la Cruz 1988. 
Margaret Sanger, parue à Londres en 1939. Dans son autobiographie, Margaret Sanger raconte sa prise de conscience féministe. Au départ, elle était actrice, elle est ensuite devenue infirmière. Conjointement à son métier d'infirmière, elle se consacrait à la lutte des femmes et plus particulièrement au droit à l'avortement.

Elle est devenue une féministe dévouée, défendant le droit à la "maternité volontaire" et se battant pour les droits des femmes pour avoir accès à l'information concernant leurs propres corps, [...] Sanger a travaillé pour libérer les femmes des grossesses non souhaitées, [...] elle a risqué l'emprisonnement en promulguant des informations à propos de l'avortement, qui était illégal à cette période ${ }^{15}$.

La lecture de l'autobiographie d'une femme, dont l'existence a changé de direction pour se consacrer à la cause féministe, a peut-être suscité chez Frida Kahlo une prise de conscience dont les œuvres produites après 1939 sont le résultat.

Autorretrato con Pelo Cortado est une image de transgression, au travers de laquelle Frida Kahlo s'exprime non seulement en son nom, mais aussi au nom de toutes les femmes soumises à leurs rôles sociaux et moraux. Une image transgressive visant à la libération des femmes. Son visage montre des signes de fatigue, mais elle reste fière et ne laisse filtrer aucun sentiment puisque son regard est impassible ; elle se montre à la fois vulnérable et forte. Face à une telle œuvre, nous sommes tiraillés entre deux sentiments: une impression de fragilité, elle est seule dans une profonde détresse, mais il règne aussi un sentiment de parfaite maitrise de soi, de force, car à la lecture des paroles de la chanson nous comprenons que l'artiste a volontairement choisi de couper ses cheveux et par là même de prendre le risque d'être rejetée par la société. Elle a choisi la rupture et l'état de solitude. Elle n'est désormais plus une victime. Son visage de marbre est un véritable masque que nous retrouvons dans la quasi-totalité de ses autoportraits. Le masque de Kahlo est le visage de toutes les femmes. L'œuvre dégage une force inouie, du fait de sa posture, de la violence du geste et de l'amoncellement des mèches de cheveux. Elle est la femme Pelona ("chauve »), alors que son visage reste insensible à l'action et à toute émotion. Le contraste entre la scène et son visage

15 Ankori $2002: 182-183$. 
confère à l'autoportrait un caractère puissant. Elle se présente au spectateur dans un état de nudité inhabituelle, dévoilant une partie de sa véritable identité, qui n'est plus déguisée, ornementée ou aidée. Un « nouveau Soi est exposé »"16.

\section{Prémices de l'art Queer}

Autorretrato con Pelo Cortado est en outre une œuvre capitale pour appréhender la question de l'identité androgyne de Frida Kahlo. L'œuvre fait allusion à une identité sexuelle ambiguë. L'autoportrait contient une tension palpable, un conflit entre les pôles masculin et féminin. Gannit Ankori estime que l'œuvre est «inhérente à sa sexualité » et qu'il faut revenir sur l'histoire de l'artiste pour comprendre son caractère androgyne ${ }^{17}$. Étant enfant, elle était considérée comme un "garçon manqué ", elle pratiquait différents sports pour se remettre musculairement d'une poliomyélite qu'elle avait contractée à l'âge de six ans. Son médecin «s'est attaché à lui faire pratiquer des activités tout à fait inhabituelles chez les jeunes filles des bonnes familles mexicaines de l'époque : le football, la boxe, la lutte et la natation $»^{18}$. L'artiste disait elle-même: "Mes jouets, étaient ceux d'un garçon : des patins à roulettes et des bicyclettes $»^{19}$. De plus, le 7 février 1926, son père, Guillermo Kahlo réalise un portrait de famille, où elle apparaît à l'âge de dix-neuf ans, les cheveux tirés en arrière, habillée en costume trois pièces et tenant une canne à la main. Tel un dandy, elle se tient aux côtés d'un oncle, sur l'épaule duquel elle place son avant-bras, conférant à ce geste une complicité masculine mutuelle. Elle semble à la fois se moquer de l'attitude virile et affirmer un aspect de sa personnalité. Si à l'époque, sa tenue et son attitude ont été considérées comme une farce, « une vue rétrospective des images, nous révèle aussi des déclarations à propos de l'ambiguïté sexuelle innée, de sa conscience du rôle des costumes dans la formation de l'identité, et de son affirmation du droit d'être différent

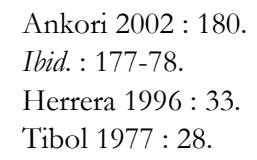


et non conventionnel $»^{20}$. L'auteur rappelle enfin qu'au sein du couple Kahlo-Rivera, ce dernier aimait le côté "garçon manqué » de sa compagne, tandis que Kahlo aimait les formes et traits féminins du corps de Rivera. En 1949, elle écrit à propos de Rivera :

En le voyant nu, on pense immédiatement à une jeune grenouille mâle dressée sur ses pattes de derrière. Sa peau est d'un blanc verdâtre identique à celle d'un animal aquatique. [...] Ses épaules juvéniles, étroites et rondes, coulent sans angles dans des bras féminins qui se terminent par des mains merveilleuses, petites et d'un dessin délicat, sensibles et subtiles comme des antennes qui communiquent avec tout l'univers ${ }^{21}$.

Une description mise en œuvre au moyen de deux Cadavres Exquis réalisés avec Lucienne Bloch à Détroit ${ }^{22}$. Le premier présente un corps de femme, avec un avant-bras dont la pilosité est accentuée, surmonté de la tête de Rivera. Le personnage tient un balai et porte des chaussures à talons hauts. Le second dessin présente une moitié de corps de femme qui semble être celui de Kahlo puisque l'on peut reconnaitre ses attributs significatifs qui sont la coiffure en chignon, les sourcils joints, le collier de perles, et le corset orthopédique. La partie basse du corps androgyne est celui de Rivera comme l'indique l'inscription en bas à droite. On y voit un sexe masculin surmonté d'une feuille de vigne qui est actionnée par les mains de Kahlo au moyen de deux ficelles, telle une marionnette. Le personnage est en train d'uriner ou d'éjaculer dans un récipient qui se situe entre ses jambes. Kahlo joue volontiers dans son œuvre avec son identité bisexuelle, en juxtaposant les éléments ou attributs des deux genres, elle crée un personnage hybride. De son vivant, elle ne cachait pas son homosexualité et partageait publiquement ses aventures

20 Ankori $2002: 178$.

21 Kahlo 1949: 38 .

22 Le Cadavre Exquis était un jeu très apprécié des surréalistes, qui se joue à plusieurs. Il s'agit de dessiner sur une partie d'une feuille de papier, chacun à son tour continue le dessin en sachant que la feuille est pliée à chaque fois pour que le nouveau participant ne voit pas l'intégralité du dessin. Le résultat est donc une somme d'imagination collective due au hasard.

Lucienne Bloch, fille du compositeur suisse Ernest Bloch, était une des nombreuses assistantes de Diego Rivera, ainsi qu'une amie proche de Frida Kahlo, elle était venue de New York pour s’installer chez les Rivera à Détroit. 
bisexuelles. Une attirance qu'elle a d'ailleurs représentée dans une peinture datée de 1939 et intitulée Dos Desnudos en un Bosque o La Tierra Misma o Mi Nana y Yo («Deux Nus dans la Forêt ou La Terre Même ou Ma Nourrice et Moi»). La scène montre deux femmes nues : une Indienne est assise au bord d'une faille terrestre, sur sa jambe se prélasse une femme métisse ; il s'agit de l'artiste elle-même. Elles sont seules entre deux mondes : la jungle luxuriante, au milieu de laquelle un singe-araignée (symbolisant l'homme voyeur) les épie discrètement, et le désert aride. Une peinture à travers laquelle elle assume et revendique sa liberté sexuelle.

La fusion des genres est flagrante dans Autorretrato con Pelo Cortado, où Frida Kahlo se présente sous les traits d'un être androgyne. Elle y associe visuellement les genres en les plaçant sur un même plan, ce qui est une des caractéristiques innovatrices pour la période concernée. L'artiste démontre avec subtilité au spectateur que la limitation des genres est oppressante et restrictive pour la recherche du Soi. À ce titre, il n'est pas étonnant que la redécouverte de sa production dans les années 1970 fut enthousiasmante pour les théoriciennes féministes qui ont mis en évidence une conscience politique et sociale inédite sur des questions qui restent actuelles. Frida Kahlo a laissé de côté la fausse pudeur pour aller à l'essentiel de son expérience corporelle, psychologique et sociale. Au moyen d'une iconographie et de problématiques subversives, elle a brisé des tabous comme la figuration du sang vaginal, la nudité féminine non idéalisée, l'expérience biologique féminine qui n'avait jamais jusque-là été représentée parce qu'elle était cantonnée à la sphère privée. En cela son œuvre est une traduction visuelle du slogan féministe scandé durant les années 1970 : «Le personnel est politique ». Sa vie et son expérience sont mises au service d'une cause plus grande.

\section{Bibliographie}

ANKORI Gannit, 2002, Imaging her selves: Frida Kablo's Poetics of Identity and Fragmentation, Westport, Greenwood Publishing Group.

BRETON André, 1965, Le Surréalisme et la Peinture, Paris, Gallimard. 
De La Cruz Juana Inès, 1988, A Sor Juana Anthology, Cambridge, Mass., Harvard University Press.

Freud Sigmund, 1951 (1 éd., 1926), Inbibition, Symptôme et Angoisse, Paris, PUF.

Herrera Hayden, 1996, Frida : Biographie de Frida Kablo, Paris, Anne Carrière.

KAHLO Frida, 1949, "Retrato de Diego", Diego Rivera. Homenaje a los 50 años de su Labor Artistica, Mexico, Palacio de Bellas Artes.

Lindauer Margaret A., 1999, Devouring Frida: The Art History and Popular Celebrity of Frida Kablo, Hanover, Wesleyan University Press.

LOwE Sarah M., 1991, Frida Kablo, New York, Universe.

Poniatowska Elena \& Carla Stellweg, 1992, Frida Kablo: The Camera Seduced, San Francisco, Chronicle Books.

Tibol Raquel, 1977, Frida Kablo: Cronica, Testimonios y Aproximaciones, Mexico, Editiones de Cultura Popular.

—, 1993, Frida Kablo: An Open Life, New Mexico, University of New Mexico Press. [1 1 re éd., Mexico, Oasis, 1983]. 